\title{
ESTUDIO DE RIESGOS ERGONÓMICOS Y CONDICIONES DE TRABAJO DECENTES EN LA AGROEXPORTACIÓN DE CAFÉ HONDUREÑO
}

\author{
STUDY OF ERGONOMIC RISKS AND DECENT WORKING CONDITIONS IN THE EXPORT \\ OF HONDURAN COFFEE AGRO
}

\section{Héctor Emilio Madrid-Casaca*}

\begin{abstract}
Resumen: La producción del café es importante a nivel mundial siendo una de las industrias con mayor relevancia en el desarrollo y funcionamiento de las naciones. Es relevante identificar los riesgos ergonómicos y condiciones de trabajo decente en la actividad de agro exportación de café hondureño. Amerita el esfuerzo de realizar la investigación y como punto de partida, lograr conocer la realidad de las vivencias de los trabajadores en el campo, y a su vez, presentar factores de riesgo en la seguridad y salud de las personas en sus puestos laborales, que si se toman las consideraciones, también puede traer beneficios en la productividad y calidad del café. Así, el artículo analiza los problemas que enfrentan las personas que trabajan en las fincas en la producción y recolección de café. Para esto se practicó una encuesta dirigida a 150 trabajadores en varias fincas en la ciudad de Marcarla, La Paz. Se revisaron estudios similares realizados en otros países tomando como base algunos puntos específicos que tenían relación con lo referente a lo que pasa en Honduras. En base a la información obtenida, se puede precisar que en su mayoría los entrevistados sí tienen conocimientos de los riesgos ergonómicos a los que se enfrentan al realizar estas labores, así como sobre las condiciones que se necesitan para llevar a cabo de una manera correcta su trabajo, y que según ellos, hasta el momento no han sido las más adecuadas.
\end{abstract}

Palabras clave: Ergonomía, trabajo decente, riesgos, agroexportación.

\begin{abstract}
Coffee production is important worldwide, being one of the industries with the greatest relevance in the development and operation of nations. It is relevant to identify ergonomic risks and decent work conditions in the Honduran coffee agro-export activity. It is worth the effort to carry out the investigation and as a starting point, to get to know the reality of the experiences of workers in the field, and in turn, present risk factors in the safety and health of people in their jobs, which if the considerations are taken, it can also bring benefits in the productivity and quality of the coffee. Thus, the article analyzes the problems faced by people who work on farms in the production and harvesting of coffee. For this, a survey was conducted with 150 workers in various farms in the city of Marcarla, La Paz. Similar studies carried out in other countries were reviewed based on some specific points that were related to what is happening in Honduras. Based on the information obtained, it can be specified that most of the interviewees do have knowledges of the ergonomic risks they face when carrying out these tasks, as well as the conditions that are needed to correctly carry out their work, and that according to them, so far have not been the most appropriate.
\end{abstract}

Keywords: Ergonomics, decent work, risks, agro-export.

*Facultad de Ciencias Económicas, Administrativas y Contables, Universidad Nacional Autónoma de Honduras. Tegucigalpa, Honduras. Correo electrónico: hector.madrid@unah.edu.hn. Orcid: https://orcid.org/oooo-0002-5486-2234 


\section{Introducción}

El café es una de las bebidas más consumidas en todo el mundo, representa el principal producto de exportación en Honduras ocupando el sexto lugar de exportación a nivel mundial en café (Catracho Global, 2018). El concepto de la ergonomía tal y como se conoce hoy en día, data de la post guerra en 1949 cuando se conforma la primera Sociedad de Investigación en Ergonomía en Gran Bretaña, y fue dicho país el primero en usar el término ergonomía, proveniente del griego Ergon: trabajo y Nomos: ley (Cloutier, 2006). En 1961 se celebró la primera reunión de la asamblea general de la Asociación Internacional de Ergonomía (IEA) en Estocolmo Suecia, esta reunión completa formalmente la fase preparatoria de la asociación y comenzó las actividades regulares. En 2011 pasó a ser una organización sin fines de lucro en Zúrich Suiza (IEA, 2020).

En un simposio en Argentina se establecen bases y contexto que amerita los esfuerzos para lograr mejores beneficios en la agricultura, donde los estudios ergonómicos buscan elevar los indicadores de productividad y calidad en los procesos, a la vez de elevar la seguridad y comodidad de los trabajadores debido a que en la mayoría de los países en vías de desarrollo las actividades agrícolas se derivan de la energía humana (Cárdenas \& Ovalle, 2017).

La perspectiva del trabajo decente puede desarrollar sentido de servicio, justicia e integridad, o por el contrario, puede deteriorar al trabajador si no encuentra aquel medio que dignifique su condición humana, lo integre en la vida social y contribuya al bien de la comunidad, cuyo enfoque es el de la economía informal, en torno a cuatro dimensiones, tales como, la promoción del empleo, el respeto de los derechos fundamentales en el trabajo, la extensión de la seguridad laboral y la protección social (Jiménez, 2011). Un estudio transversal en un grupo de recolectores de café en el municipio de Andrés, Antioquia en Colombia, demostró factores de riesgo posturales y desórdenes musculoesqueléticos, siendo la lumbalgia y cervicalgia, las repercusiones más comunes en la salud que tenían los trabajadores (Garzón-Duque et al., 2017).

La meta de la ciencia de la ergonomía es encontrar una mejor correspondencia entre el trabajador y las condiciones de trabajo. La ergonomía examina la capacidad física del cuerpo humano, y las limitaciones del cuerpo humano; en relación con las tareas que debe realizar una persona, y las herramientas utilizadas, además de su entorno de trabajo (Instituto Nacional para la Seguridad y Salud Ocupacional [NIOSH], 2001). La agricultura es un trabajo de mucho esfuerzo, y los trabajadores sufren lesiones y dolores de espalda, de brazos y manos, más que cualquier otro problema de salud. Estas lesiones son las causas más comunes de incapacidad, pero en Honduras, no hay ente regulador de la salud de los trabajadores agrícolas, ni una organización que capacite al personal agrícola al momento de realizar sus labores.

El tema de la ergonomía, trabajo decente y su aplicación en el sector agroindustrial a 
nivel mundial manifiesta un creciente interés, por el trabajo en el desarrollo de las producciones, sin embargo, hoy en día la Organización de las Naciones Unidas para la Alimentación y la Agricultura (FAO), define las condiciones de trabajo en las zonas rurales, de manera que tienden a ser difíciles, precarias y peligrosas puesto que, los empleos rurales son generalmente informales, no existen contratos escritos y los empleados no gozan de protección social (FAO, 2020).

El Instituto Hondureño del café (IHCAFE), tiene como objetivo brindar asistencia técnica y transferencia de nuevas tecnologías desarrolladas por la institución a más de 100 mil productores de café. Un equipo técnico conformado por más de 100 técnicos en las ciencias agrícolas con amplia experiencia y capacidad transfieren las tecnologías y conocimientos por medio de: visitas y diagnósticos de finca, días de campo, giras técnicas, días demostrativos, establecimiento y manejo de parcelas. El IHCAFE cuenta con 7 oficinas regionales y 46 agencias de extensión estratégicamente localizadas en las áreas productoras de café.

En estudios sobre el tema a nivel internacional, se han estado llevando a cabo varios análisis para poder identificar cuáles son los principales riesgos ergonómicos a los cuales están expuestos los trabajadores en la producción del campo a nivel de América Central y América del sur, y poder ayudar a mejorar sus condiciones de trabajo para disminuir los problemas que estos conllevan. Algunas de las entidades que se encargan de realizar estudios relacionados a los riesgos ergonómicos son: el Programa Salud, Trabajo y Ambiente (SALTRA), el Instituto Hondureño de Seguridad Social (IHSS), la Secretaría de Salud, entre otros.

Es por ello que, se determinó como objetivo en la presente investigación, identificar los riesgos ergonómicos asociados y condiciones de trabajo decente en la actividad de agroexportación de café hondureño, y la manera de cómo afecta la salud de las personas y las condiciones en que trabajan, partiendo del conocimiento o no de los riesgos ergonómicos y condiciones de trabajo.

\section{Materiales y métodos}

Esta investigación se desarrolló bajo un enfoque cualitativo, basado en métodos de recolección y análisis de los datos no estandarizados ni predeterminados. El tipo de muestreo seleccionado fue el no probabilístico por conveniencia, estas muestras están formadas por los casos disponibles a los cuales se tienen acceso, esto es porque no se puede acceder a las regiones cafetaleras, por ende, se ha determinado establecer cercanía con algunas personas que han estado en el trabajo cafetalero, para poder obtener información. La gran mayoría del café producido en Honduras proviene de regiones montañosas de 210 de los 298 municipios y 15 de los 18 departamentos del país, generando más de un millón de trabajos que producen cerca del 38\% del PIB Agrícola (IHCAFE, 2018).

Debido a la pandemia por COVID-19, además de los huracanes ETA e IOTA, fenómenos naturales acaecidos en Honduras a finales del 2020, la fuente de información primaria a la 
que se recurrió fue la encuesta aplicada a productores, recolectores y personas con conocimientos en lo referente a la producción de café. Se hizo énfasis a una encuesta dirigida a 150 trabajadores en varias fincas en la ciudad de Marcarla, La Paz, los cuales están organizados por una empresa hondureña con certificaciones de Fairtrade, UTZ certified, denominación de origen Café Marcala, Manos de Mujer y certificación orgánica. La empresa es estable y creciente con más de 20 compradores en los países de Estados Unidos, Canadá, Alemania, Bélgica, Dinamarca, Inglaterra, Irlanda, Italia, Japón y Corea del Sur.

Se revisaron estudios similares realizados en otros países tomando como base algunos puntos específicos que tenían relación con lo referente a lo que pasa en Honduras, realizando una comparación previa de la información relevante de otros estudios relacionados. Debido a que en Honduras no se han realizado estudios específicos sobre los riesgos ergonómicos, resulta difícil medir la cantidad de personas que sufren algún problema de salud al participar en la producción de café, ya que estas al sufrir algún inconveniente, en su mayoría deciden asistir personalmente a recibir atención médica sin informar a sus patronos o personas responsables de la producción del café.

\section{Resultados y discusión}

En la tabla 1 se presenta información sobre las principales regiones del país, en la cual se destacan las variedades de café, las características de taza, altura sobre el nivel del mar, entre otras, aspectos que son considerados por los expertos para la inversión en la fruta, especialmente para exportación a diversas partes del mundo.

Como se puede ver, en la región de Montecillos, en la ubicación geografía, destaca La Paz, donde se encuentra el municipio de Marcala, en el cual se desarrolló el instrumento de la presente investigación. Importante mencionar que se encuentra a una altura de entre 1.200 a 1.600 metros sobre el nivel del mar, ideal para la producción de café de alta calidad.

La cosecha y recolección de la fruta en estas regiones de Honduras, se realiza entre los meses de noviembre a marzo de cada año, a lo que popularmente se le denomina la temporada.

Tabla 1. Información por región en Honduras.

\begin{tabular}{|c|c|c|c|c|c|c|c|}
\hline REGIÓN & $\begin{array}{l}\text { Características } \\
\text { de taza }\end{array}$ & $\begin{array}{l}\text { Ubicación } \\
\text { geográfica }\end{array}$ & $\begin{array}{l}\text { Altura } \\
\text { (MSNM) }\end{array}$ & $\begin{array}{l}\text { Precipitación } \\
\text { anual (MM) }\end{array}$ & $\begin{array}{l}\text { Temperatura } \\
{ }^{\circ} \mathrm{C}\end{array}$ & $\begin{array}{l}\text { Época de } \\
\text { cosecha }\end{array}$ & Variedades \\
\hline $\begin{array}{l}\text { Región de } \\
\text { Copan }\end{array}$ & $\begin{array}{l}\text { Café con sabor a } \\
\text { chocolate, } \\
\text { cuerpo redondo, } \\
\text { bien balanceado, } \\
\text { con un post } \\
\text { gusto sostenido. }\end{array}$ & $\begin{array}{l}\text { Copán, } \\
\text { Ocotepeque, } \\
\text { parte de } \\
\text { Lempira, } \\
\text { Cortés y } \\
\text { Santa } \\
\text { Bárbara. }\end{array}$ & $\begin{array}{l}1.000- \\
1.500\end{array}$ & $1.300-2.300$ & $11,5-22,3$ & $\begin{array}{l}\text { Noviembre } \\
\text { a marzo }\end{array}$ & $\begin{array}{l}\text { Bourbon, } \\
\text { Caturra, } \\
\text { Catuai }\end{array}$ \\
\hline $\begin{array}{l}\text { Región de } \\
\text { Opalaca }\end{array}$ & $\begin{array}{l}\text { Un café con una } \\
\text { fina y delicada } \\
\text { acidez, bien } \\
\text { balanceado con } \\
\text { sabores a frutas }\end{array}$ & $\begin{array}{l}\text { Santa } \\
\text { Bárbara, } \\
\text { Intibucá y } \\
\text { Lempira }\end{array}$ & $\begin{array}{l}1.100^{-} \\
1.500\end{array}$ & $1.400-1.950$ & $14,2-21,4$ & $\begin{array}{l}\text { Noviembre } \\
\text { a febrero }\end{array}$ & $\begin{array}{l}\text { Bourbon, } \\
\text { Catuai, } \\
\text { Typica }\end{array}$ \\
\hline
\end{tabular}




\begin{tabular}{|c|c|c|c|c|c|c|c|}
\hline & $\begin{array}{lr}\text { tropicales } r \text { tales } \\
\text { como uvas y } \\
\text { moras, post } \\
\text { gusto dulce y } \\
\text { cuerpo plano. } \\
\end{array}$ & & & & & & \\
\hline $\begin{array}{l}\text { Región de } \\
\text { Montecillos }\end{array}$ & $\begin{array}{l}\text { Un café con } \\
\text { fragancias } \\
\text { frutales y dulces, } \\
\text { su acidez es viva } \\
\text { y brillante, con } \\
\text { un sabor a } \\
\text { naranja y } \\
\text { durazno, creando } \\
\text { una bebida } \\
\text { vibrante, con un } \\
\text { post gusto } \\
\text { entonante y un } \\
\text { cuerpo } \\
\text { aterciopelado. }\end{array}$ & $\begin{array}{l}\text { La Paz, } \\
\text { Comayagua, } \\
\text { Santa } \\
\text { Bárbara e } \\
\text { Intibucá. }\end{array}$ & $\begin{array}{l}1.200- \\
1.600\end{array}$ & $1.300-2.300$ & $12,0-21,2$ & $\begin{array}{l}\text { Diciembre } \\
\text { a abril }\end{array}$ & $\begin{array}{l}\text { Bourbon, } \\
\text { Catuai, } \\
\text { Caturra, } \\
\text { Pacas }\end{array}$ \\
\hline $\begin{array}{l}\text { Región de } \\
\text { Comayagua }\end{array}$ & $\begin{array}{l}\text { Un café con una } \\
\text { dulce fragancia } \\
\text { cítrica, su acidez } \\
\text { es cítrica } \\
\text { vibrante con } \\
\text { sabores dulces y } \\
\text { a chocolate y un } \\
\text { cuerpo cremoso. }\end{array}$ & $\begin{array}{l}\text { Comayagua, } \\
\text { Francisco } \\
\text { Morazán }\end{array}$ & $\begin{array}{l}1.000- \\
1.500\end{array}$ & $1.350-1.700$ & $14,0-22,0$ & $\begin{array}{l}\text { Diciembre } \\
\text { a marzo }\end{array}$ & $\begin{array}{l}\text { Bourbon, } \\
\text { Caturra y } \\
\text { Typica }\end{array}$ \\
\hline $\begin{array}{l}\text { Región de } \\
\text { El Paraíso }\end{array}$ & $\begin{array}{l}\text { Café con sabores } \\
\text { cítricos, con una } \\
\text { fragancia dulce, } \\
\text { cuerpo suave y } \\
\text { una fina acidez, } \\
\text { con un post } \\
\text { gusto muy } \\
\text { sostenido. }\end{array}$ & $\begin{array}{l}\text { El Paraíso y } \\
\text { parte de } \\
\text { Choluteca y } \\
\text { Olancho }\end{array}$ & $\begin{array}{l}1.000^{-} \\
1.400\end{array}$ & $950-1.950$ & $16,0-22,5$ & $\begin{array}{l}\text { Diciembre } \\
\text { a marzo }\end{array}$ & $\begin{array}{ll}\text { Catuai } & y \\
\text { Caturra } & \end{array}$ \\
\hline $\begin{array}{l}\text { Región de } \\
\text { Agalta }\end{array}$ & $\begin{array}{lr}\text { Café con diversos } \\
\text { sabores a frutas } \\
\text { tropicales } & \text { con } \\
\text { fragancia } & \text { a } \\
\text { caramelo } & \text { y } \\
\text { chocolate y } & \text { post } \\
\text { gusto dulce, } & \text { su } \\
\text { acidez } & \text { es } \\
\text { pronunciada. }\end{array}$ & $\begin{array}{ll}\text { Olancho } & y \\
\text { Yoro } & \end{array}$ & $\begin{array}{l}1.000- \\
1.400\end{array}$ & $1.300-1.950$ & $14,5^{-22,5}$ & $\begin{array}{l}\text { Diciembre } \\
\text { a marzo }\end{array}$ & $\begin{array}{l}\text { Bourbon, } \\
\text { Caturra } \\
\text { Typica }\end{array}$ \\
\hline
\end{tabular}

Nota. Adaptado de "Las regiones cafetaleras de Honduras", IHCAFE.

(https://www.ihcafe.hn/regiones-cafetaleras/). 
En la tabla 2, se muestra que de las 150 personas encuestadas, 115 corresponden al sexo masculino y 35 al femenino.

Tabla 2. Personas encuestadas según el sexo.

\begin{tabular}{|l|c|}
\hline Sexo & Personas \\
\hline Masculino & 115 \\
\hline Femenino & 35 \\
\hline Total & $\mathbf{1 5 0}$ \\
\hline
\end{tabular}

La tabla 3 muestra las bandas por edades de las personas del sexo masculino entrevistadas, en la cual el mayor porcentaje, de un $28 \%$, corresponde a las edades de entre 15 y 25 años, seguido de edades entre 36 y 45, y el menor porcentaje, de un 8\%, a personas mayores de 66 años.

Tabla 3. Bandas por edades personas entrevistadas del sexo masculino.

\begin{tabular}{|l|c|c|}
\hline \multicolumn{3}{|c|}{ Edad laboral sexo masculino } \\
\hline Edad & Personas & Porcentaje \\
\hline Entre 15 y 25 & 32 & $28 \%$ \\
\hline Entre 26 y 35 & 26 & $23 \%$ \\
\hline Entre 36 y 45 & 23 & $20 \%$ \\
\hline Entre 46 y 55 & 13 & $11 \%$ \\
\hline Entre 56 y 65 & 12 & $10 \%$ \\
\hline Mayor de 66 & 9 & $8 \%$ \\
\hline Total & $\mathbf{1 1 5}$ & $\mathbf{1 0 0 \%}$ \\
\hline
\end{tabular}

La tabla 4 muestra la edad de los trabajadores del sexo femenino, en la cual el mayor porcentaje, de un $26 \%$, corresponde a las edades de entre 15 y 25 años y entre 46 y 55 años, y el menor porcentaje, de un 6\%, a personas mayores de 66 años.

Tabla 4. Bandas por edades personas entrevistadas del sexo femenino.

\begin{tabular}{|l|c|c|}
\hline \multicolumn{3}{|c|}{ Edad laboral sexo femenino } \\
\hline Edad & Personas & Porcentaje \\
\hline Entre 15 y 25 & 9 & $26 \%$ \\
\hline Entre 26 y 35 & 7 & $20 \%$ \\
\hline Entre 36 y 45 & 5 & $14 \%$ \\
\hline Entre 46 y 55 & 9 & $26 \%$ \\
\hline Entre 56 y 65 & 3 & $8 \%$ \\
\hline Mayor de 66 & 2 & $6 \%$ \\
\hline Total & $\mathbf{3 5}$ & $\mathbf{1 0 0 \%}$ \\
\hline
\end{tabular}

La tabla 5 muestra los problemas de salud de los trabajadores producto del trabajo realizado en diferentes posturas, en la cual se observa que, el 29\% de los trabajadores 
presentan dolores de espalda, seguido de dolor en las articulaciones con un $25 \%$, y la fatiga, en menor porcentaje, con un $8 \%$.

Tabla 5. Problemas de salud de los trabajadores.

\begin{tabular}{|l|c|c|}
\hline \multicolumn{3}{|c|}{ Problemas de salud de las personas } \\
\hline Malestar & Total & Porcentaje \\
\hline Dolor de espalda & 43 & $29 \%$ \\
\hline Dolor de articulaciones & 37 & $25 \%$ \\
\hline Dolor de pies & 36 & $24 \%$ \\
\hline Dolor de cuello & 22 & $15 \%$ \\
\hline Fatiga & 12 & $8 \%$ \\
\hline Total & $\mathbf{1 5 0}$ & $\mathbf{1 0 0 \%}$ \\
\hline
\end{tabular}

La tabla 6 muestra el porcentaje de herramientas más utilizadas en la producción, con un $35 \%$, el uso de la barra, seguido con un $24 \%$, la piocha. Es importante mencionar que, todas las herramientas descritas en la tabla son extremadamente peligrosas debido a que son corto punzantes.

Tabla 6. Herramientas utilizadas en producción de café.

\begin{tabular}{|l|c|c|}
\hline \multicolumn{1}{|c|}{ Herramientas } & Número de personas que la utilizan & Porcentaje \\
\hline Barra & 52 & $35 \%$ \\
\hline Piocha & 36 & $24 \%$ \\
\hline Azadón & 28 & $19 \%$ \\
\hline Machete & 19 & $13 \%$ \\
\hline Pujaguante & 15 & $10 \%$ \\
\hline \multicolumn{1}{|c|}{ Total } & $\mathbf{1 5 0}$ & $\mathbf{1 0 0 \%}$ \\
\hline
\end{tabular}

La tabla 7 muestra el factor ergonómico asociado a la carga estática, producto de la mantención de la postura que adoptan los trabajadores al momento de ejercer sus labores, las cuales, la mayoría las realizan de pie.

Tabla 7. Factor ergonómico carga estática.

\begin{tabular}{|l|c|c|}
\hline \multicolumn{3}{|c|}{ Carga estática } \\
\hline Posición & Personas & Porcentaje \\
\hline De pie & 67 & $45 \%$ \\
\hline Sentado & 41 & $27 \%$ \\
\hline De rodillas & 23 & $15 \%$ \\
\hline Encorvado & 19 & $13 \%$ \\
\hline \multicolumn{1}{|c|}{ Total } & $\mathbf{1 5 0}$ & $\mathbf{1 0 0 \%}$ \\
\hline
\end{tabular}




\section{Conclusiones}

Las personas que trabajan actualmente en la agricultura pueden estar expuesta a riesgos ergonómicos, con la probabilidad de desarrollar trastornos, afecciones o enfermedades que involucran a los tendones, músculos, nervios y otras estructuras que dan soporte y estabilidad al cuerpo humano, ocasionados por los requerimientos físicos en el trabajo, fundamentalmente debido a la adopción de posturas forzadas, la realización de movimientos repetitivos, la manipulación manual de cargas y la aplicación de fuerzas.

Esta investigación refleja los principales efectos en la salud, provocados a causa de la postura que adoptan los trabajadores para recolectar el café. Esto produce dolores de espalda, además de dolores musculares, entre otros. Igualmente, están sujetos a complicaciones de salud por estar expuestos a fertilizantes y pesticidas aplicados a las plantaciones. Además de estos riesgos ergonómicos, la topografía de los terrenos puede llegar a ocasionar caídas o resbalarse al caminar lo que podría ocasionar lesiones.

Según los productores y representantes de diversas organizaciones gremiales, el gobierno debería ayudar a los pequeños y medianos caficultores, en la creación de espacios con toda la infraestructura que se requiere para llevar a cabo el proceso de la producción de café, ya que algunos que no tienen disposición de recursos, se ven obligados a vender el café a menor precio.

Otra situación de interés nacional, y especialmente en las zonas productoras de café, es priorizar recursos económicos y políticas públicas para apoyar en el mantenimiento y reparación de la infraestructura vial, así como de las carreteras internas que conducen hacia las fincas de café, esto para que el ente que realiza la compra directa al caficultor, no lo haga incurrir en gastos de flete por el mal estado de las infraestructuras viales. De esta manera, se obtendrían beneficios económicos que pueden favorecer a los miles de productores del aromático.

Es importante mencionar que, en base a la información obtenida, se puede precisar que en su mayoría los entrevistados sí tienen conocimientos de los riesgos ergonómicos a los que se enfrentan al realizar estas labores, y de las condiciones necesarias que estos necesitan para llevar a cabo bien su trabajo, y que, según ellos, hasta el momento no han sido las más adecuadas. 


\section{Referencias}

Asociación Internacional de Ergonomía. (2020). History of the IEA. https://iea.cc/history/

Cárdenas, D., \& Ovalle, A. (2017). Décimo simposio internacional de ingeniería industrial: Actualidad y nuevas tendencias. Repositorio Institucional Digital de la Universidad Nacional de Misiones (RIDUNAM). https://hdl.handle.net/20.500.12219/2818

Catracho Global. (2018). El café de Honduras. http://catrachoglobal.com/politica/el-cafe-dehonduras/

Cloutier, E. (2006). Plusieurs aspects importants permettant d'aborder la santé des personnes au travail. https://doi.org/10.4000/pistes.3057

Garzón-Duque, M. O., Vásquez-Trespalacios, E. M., Molina-Vásquez, J., \& Muñoz-Gómez, S. G. (2017). Condiciones de trabajo, riesgos ergonómicos y presencia de desórdenes músculoesqueléticos en recolectores de café de un municipio de Colombia. Revista de la Asociación Española de Especialistas en Medicina del Trabajo, 26(2), 127-136. https://scielo.isciii.es/pdf/medtra/v26n2/1132-6255-medtra-26-02-00127.pdf

Instituto Hondureño del café. (2018). Regiones cafetaleras. https://www.ihcafe.hn/regionescafetaleras/\#region-montecillos

Instituto Nacional para la Seguridad y Salud Ocupacional. (2001). Soluciones simples: Ergonomía para trabajadores agrícolas. https://www.cdc.gov/spanish/niosh/docs/2001$111 \mathrm{sp} / \mathrm{pdfs} / 2001-111 \mathrm{sp} . \mathrm{pdf}$

Jiménez, M. (2011). La economía informal y el mercado laboral. Un análisis desde la perspectiva del trabajo decente. Universidad Nacional de La Plata. https://www.cedlas.econo.unlp.edu.ar/wp/wp-content/uploads/doc cedlas116.pdf

Organización de las Naciones Unidas para la Alimentación y la Agricultura. (2020). Condiciones de trabajo. http://www.fao.org/rural-employment/work-areas/working-conditions/es/ 